Research Article

Animal Genetics

\title{
Transcriptome analyses of Ditylenchus destructor in responses to cold and desiccation stress
}

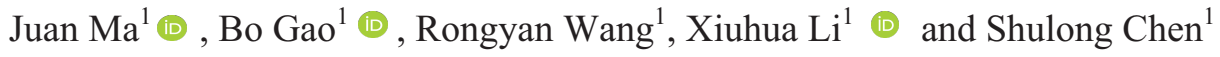 \\ ${ }^{1}$ Institute of Plant Protection, Hebei Academy of Agricultural and Forestry Sciences /IPM centre of Hebei \\ Province/ Key Laboratory of Integrated Pest Management on Crops in Northern Region of North China, \\ Ministry of Agriculture, Baoding, China.
}

\begin{abstract}
The objective of this study was to identify molecular responses in Ditylenchus destructor to cold and desiccation by means of transcriptomes analyses. A total of 102,517 unigenes were obtained, with an average length of $1,076 \mathrm{bp}$, in which $58,453(57 \%)$ had a functional annotation. A total of 1154 simple sequence repeats (SSRs) distributed over 1078 unigenes were detected. Gene expression profiles in response to cold and desiccation stress and the expression of specific stress-related genes were compared. Gene ontology analysis and pathway-based analysis were used to further investigate the functions of the differentially expressed genes. The reliability of the sequencing data was verified through quantitative real-time PCR analysis of 19 stress-related genes. RNA interference used to further assess the functions of the cold-related unigenes 15628 and 15596 showed that the knockdown of each of these genes led to decreased cold tolerance of $D$. destructor. Hence, this study revealed molecular processes and pathways active in cold- or dessication-treated nematodes. The transcriptome profiles presented in this study provide insight into the transcriptome complexity and will contribute to further understand stress tolerance in $D$. destructor.
\end{abstract}

Keywords: Potato rot nematode, transcriptome, tolerance, differentially expressed genes.

Received: March 01, 2018; Accepted: May 03, 2019.

\section{Introduction}

The potato rot nematode Ditylenchus destructor has become a serious problem to agriculture worldwide, mostly in temperate regions (EPPO, 2018). In addition to potato, $D$. destructor may feed on more than 100 species of plants, and on the mycelia of over 65 species of soil-inhabiting fungi (Sturhan and Brzeski, 1991). China accounts for the highest sweetpotato (Ipomoea batatas (L.) Lam.) production in the world (FAOSTAT, 2016) and sweetpotato is the main D. destructor host in China. The nematode damages sweetpotato roots, sprout, stem and vine (Qi et al., 2008). As a migratory endoparasite, all stages of $D$. destructor can be found either in the host plant tissues or in the surrounding soil. It can continue to live and reproduce within tuberous roots in storage. Once established, $D$. destructor is difficult to eliminate from the field. Sweetpotato yield losses due to $D$. destructor damage range from $10 \%$ to $30 \%$, and up to $100 \%$ under heavy infestations (Wang et al., 2018). Ditylenchus destructor is particularly widespread in north China (Qi et al., 2008), which features a temperate climate with very cold and dry winters. According to our own observation data in Baoding, Hebei, China from 2015 to 2018, the average soil temperature at $10 \mathrm{~cm}$ depth below the soil surface is -1 to $-3{ }^{\circ} \mathrm{C}$ in

Send correspondence to Shulong Chen. Institute of Plant Protection, Hebei Academy of Agricultural and Forestry Sciences, 437 Dongguan Street, Baoding 071000, Hebei, China. E-mail: chenshulong63@163.com.
January. Despite the harsh conditions, this nematode could be extracted from infected sweetpotatoes which were abandoned in the field after a cold winter and can also be isolated from completely dry sweetpotatoes (data not shown). Ditylenchus destructor may have adaptation mechanisms in response to prolonged cold and desiccation pressure.

Molecular mechanisms involved in nematode cold or desiccation tolerance are far from fully understood. Freeze avoidance and freezing tolerance are the main cold tolerance strategies by which nematodes can survive cold (Wharton, 2011). The Antarctic nematode Panagrolaimus davidi expresses the recrystallization-inhibition protein (RIP) that inhibits recrystallization but has little thermal hysteresis activity (Wharton et al., 2005; Adhikari and Adams, 2011), this protein is relatively heat stable and present at low concentrations. The Antarctic soil nematode Plectus murrayi survives low temperatures by either freezing tolerance or cryoprotective dehydration (Wharton and Raymond, 2015). Ohta et al. (2014) reported that the nervous system and intestine regulate the cold tolerance of Caenorhabditis elegans, where temperature is sensed by a thermo-sensing neuron that induces insulin secretion. Trehalose plays an important role in nematode cold tolerance strategies; it depresses the supercooling point of a solution and assists cold tolerance by freeze avoidance (Ring and Danks, 1998). The free-living nematode Aphelenchus avenae accumulated large amounts of the disaccharide trehalose, late embryogenesis abundant 
(LEA) protein and anhydrin protein following exposure to a moderate reduction in relative humidity (Goyal et al., 2005). Trehalose metabolism genes were reported to play important role in hypertonic osmotic pressure in the rice white tip nematode Aphelenchoides besseyi (Chen et al., 2017). Antifreeze proteins (AFPs) can prevent ice nucleation by producing a thermal hysteresis, thus play an important role in freeze tolerance in nematodes (Adhikari et al., 2010; Ali and Wharton, 2016). Heat shock proteins (Hsps) are induced by various stressors in many nematodes (Adhikari et al., 2009; Wharton, 2011); for instance, small HSPs are essential for desiccation tolerance of C. elegans (Erkut et al., 2013). LEA proteins are widespread to protect cells against water loss and they are important for anhydrobiosis in both animals and plants (Wharton, 2011). Silencing the expression of a LEA gene in C. elegans led to reduced survival of desiccation and heat stress (Gal et al., 2004); LEA protein 3 production was elevated in infective juveniles of the entomopathogenic nematode Steinernema feltiae during desiccation stress (Gal et al., 2001). Expression of DAF-16 target genes in $C$. elegans promotes tolerance to low temperatures (Savory et al., 2011).

Transcriptome analyses of other species have contributed to the understanding of the molecular mechanisms involved in cold or desiccation stress responses (Adhikari et al., 2010; Thorne et al., 2014; Yaari et al., 2016). However, cold and desiccation abilities vary between different nematode species and involve several mechanisms. vilponis et al. (2011) examined the cold tolerance of $D$. destructor in various aqueous environments as well as in potato tissues. After $24 \mathrm{~h}$ exposure at $-5^{\circ} \mathrm{C}, \mathrm{D}$. destructor mortality in water and gravel was significantly higher than in potato and M9 buffer. Nematode infested potato tissues were then exposed to -5 , $-8,-18$ and $-30{ }^{\circ} \mathrm{C}$ for $24 \mathrm{~h}$, and the result showed that the younger juveniles are significantly more tolerant than adults and fourth-stage juveniles. Peng et al. (2013) generated 9800 ESTs from a $D$. destructor mixed-stage library Twenty two sequences were similar to published nematode effectors, and 391 secretome members were identified from these ESTs. Resistance to cold and desiccation stress in the field is important to $D$. destructor survival, although limited information on the genes acting in these processes is available. Here we report a transcriptome analysis of gene expression patterns during the adaptation to cold and desiccation stress.

\section{Materials and Methods}

\section{Nematode culture and sample preparation}

Ditylenchus destructor was originally isolated from diseased sweetpotato in Yixian, Hebei, China (N39.35, E115.48) and has been maintained on sweetpotato $\left(25^{\circ} \mathrm{C}\right.$, $70-80 \%$ relative humidity) for more than one year. The average annual precipitation in Yixian is $538 \mathrm{~mm}$ in the last decade and is concentrated mostly in summer. Soil texture is mainly sandy loam. Mixed stages of $D$. destructor were collected from infected sweetpotato by the modified Baermann funnel method 30 days after inoculation (Wang et al., 2016).
The extracted nematodes were cleaned with sterile distilled water. Nematodes maintained under the same conditions for the same time were used for all the experiments.

\section{RNA extraction and RNA-seq library construction}

Our previous experiments indicated that the mortalities of $D$. destructor significantly increase after 35 days exposure to $10 \%$ glycerol solution or 5 days exposure to $20 \%$. To avoid death, four nematode treatments were set up. The cold stress group nematodes were exposed to water at $-1{ }^{\circ} \mathrm{C}$ for $35 \mathrm{~d}$ (D_1W) and $-3{ }^{\circ} \mathrm{C}$ for $35 \mathrm{~d}$ (D_2W). The desiccation stress group nematodes were exposed to $10 \%$ glycerol for 35 days (D_1G) and 20\% glycerol for five days (D_2G). The control group consisted of non-treated nematodes from the same batch as the desiccated and cold treated counterparts, kept in distilled water at $25^{\circ} \mathrm{C}$ for 35 days (CK_6). For each sample, millions of nematodes $(\sim 200 \mathrm{mg})$ were pooled and immediately snap frozen with liquid nitrogen and then stored at $-80{ }^{\circ} \mathrm{C}$ until used for RNA extraction.

Total RNA was extracted individually from each sample using Trizol reagent (Invitrogen, Life Technologies, USA). The RNA was subsequently treated with RNase free DNase I (Invitrogen, USA) to remove DNA contamination according to the manufacturer's protocol. RNA integrity was assessed via an Agilent 2100 Bioanalyzer (Agilent, Santa Clara, CA, USA). RNA samples with high purity and high integrity (RNA Integrity Number $\geq 9.0$ ) were used for cDNA library construction. The experimental and control groups consisted of three biologically independent RNA preparations each. RNA from the three replicates of each sample was combined for RNA-seq. The RNA-seq libraries were constructed following the manufacturer's instructions (Illumina Inc, San Diego, CA, USA). In brief, poly(A) mRNA was purified from $6 \mu \mathrm{g}$ total RNA using oligo(dT) magnetic beads, followed by fragmentation of the mRNA with fragmentation buffer. The cleaved RNA fragments served as template for first strand cDNA synthesis using reverse transcriptase and random hexamers. Second strand cDNA synthesis was done using DNA polymerase I and RNaseH. The double stranded cDNA was end-repaired and ligated to adaptors after adding a single " $\mathrm{A}$ " at the 3 ' end. The fragments with a size suitable for sequencing were isolated from the gels and enriched by PCR amplification to construct the final cDNA library. Finally, the cDNA library was sequenced on an Illumina HiSeq ${ }^{\mathrm{TM}} 4000$ platform (BGI-tech, Shenzhen, China). Raw sequencing data were submitted to NCBI Sequence Read Archive under accession number SRR5234500-SRR5234504.

\section{De novo transcriptome assembly and functional annotation}

The raw reads were cleaned by removing the adaptor sequences, poly-N and low quality sequences. The quality reads were de novo assembled into transcripts using Trinity v2.0.6, with an optimized K-mer length of 25 and all other parameters set to default (Haas et al., 2013). TGICL v2.0.6 was used to cluster transcripts to unigenes (Pertea et al., 
2003). The unigenes were annotated by aligning with the deposited ones in databases, such as NCBI non-redundant protein (Nr), Gene Ontology (GO), Swiss-Prot protein database, Kyoto Encyclopedia of Genes and Genomes (KEGG) pathway database, Cluster of Orthologous Groups (COG) database, and Interpro using BLASTX $\left(\right.$ E-value $\left.\leq 1 \mathrm{e}^{-5}\right)$. Sequence orientations and the protein coding region sequence (CDS) of unigenes were determined according to the best hit in the database. ESTScan (Iseli et al., 1999) was used to predict the sequence direction and CDS when unigenes were unaligned to any of the databases. With $\mathrm{Nr}$ annotation, the Blast2GO program (v2.5.0) with default parameters was used to obtain GO annotation of the unigenes (Conesa et al., 2005). After GO annotation for every unigene, WEGO software (Ye et al., 2006) was used for GO functional classification and graphical representation of GO terms (Zhang et al., 2017). InterProScan5 was used for InterPro annotation (Quevillon et al., 2005). Blast results information was used to extract CDS from unigene sequences and translate them into peptide sequences. To identify potential novel genes, non-annotated unigenes were predicted by ESTScan and translated into peptide sequences.

\section{Screening for SSRs and SNPS}

Potential simple sequence repeats (SSRs) marks were searched by the software MicroSatellite (http://pgrc.ipkgatersleben.de/misa/). Six types of SSRs with repeats of one to six nucleotides in length were investigated. The parameters were set to identify mono-, di-, tri-, tetra-, penta-, and hexa-nucleotide motifs with a minimum of twelve, six, five, five, four, and four repeats, respectively. The maximal distance was 100 nucleotides interrupting two adjacent distinct SSRs in a compound microsatellite. After assembly, all clean reads were mapped to unigenes using HISAT (Kim et al., 2015). The assembled contigs were scanned for single nucleotide polymorphisms (SNPs) with GATK (McKenna et al., 2010). The final SNPs were obtained after filtering out unreliable sites.

\section{Differentially expressed genes}

Clean reads were mapped to unigenes using Bowtie2 (Langmead and Salzberg, 2012), and gene expression levels were calculated by RSEM (Li and Dewey, 2011). Reads per kilo base of transcript sequence per million mapped reads (RPKM) for each unigene were calculated for gene expression profiles (Mortazavi et al., 2008). The False Discovery Rate (FDR) was calculated by the Benjamini and Hochberg's algorithm (Benjamini and Yekutieli, 2001). Differentially expressed genes between two groups were identified with defaulted filtering criteria of FDR $\leq 0.001$ and the absolute value of fold changes $\left(\log _{2}\right.$ Ratio) $\geq 2$ (Wang et al., 2010). Pairwise and multi-condition analyses were used to detect differentially expressed genes (DEGs) between two samples and among the four different treated nematode samples. For the functional and pathway enrichment analysis, the DEGs were then mapped to GO terms and the KEGG database. Significantly enriched GO and KEGG terms were determined by corrected $P \leq 0.05$. Hierarchical cluster analysis for the DEGs was performed with the pheatmap R package v1.0.8 (http://CRAN.R-project.org/package $=$ pheatmap).

\section{Quantitative Real-Time PCR validation}

Nineteen differentially expressed genes involved in the cold and dessication tolerance of $D$. destructor were randomly selected for quantitative real-time PCR (qPCR) analysis. Specific primers of selected genes were designed by Primer Premier 5.0 (Table S1). Total RNA of the control and treated nematodes was extracted separately as described above and reverse transcribed to first-strand cDNA using reverse transcriptase (TaKaRa). Real-time PCR assays were performed on an ABI Step-One Plus Real Time PCR System (Applied Biosystems) using the KAPA SYBR fast qPCR kit (Kapa Biosystems, Woburn, MA, USA) according to the manufacturer's protocol in a total volume of $20 \mu \mathrm{L}$, containing $1 \mu \mathrm{L}$ of cDNA, $0.4 \mu \mathrm{L}$ of $10 \mu \mathrm{M}$ of each primer, and 10 $\mu \mathrm{L}$ 2SYBR qPCR mixture. The PCR was performed at $95^{\circ} \mathrm{C}$ for $5 \mathrm{~min}$, followed by 40 cycles of $95^{\circ} \mathrm{C}$ for $15 \mathrm{~s}, 60^{\circ} \mathrm{C}$ for $30 \mathrm{~s}$. $\beta$-actin was used as reference gene. qPCR was carried out with three biological replicates and three technical replicates. Melting curve analysis was conducted from 60 to 95 ${ }^{\circ} \mathrm{C}$. Each of the two primer pairs for the tested genes and endogenous references produced a single peak in the melting curve analyses. Relative gene expression was calculated by the $2^{-\Delta \Delta C t}$ method (Pfaffl, 2001). The significant difference of $2^{-\Delta \Delta \mathrm{Ct}}$ among the different treatments of $D$. destructor was analyzed using the Student's $t$-test with SPSS Statistics 16.0.

\section{RNA interference analysis}

Two highly expressed genes (unigene 15596 and 15628) revealed in the RNA-seq experiments of cold treated nematodes were selected for RNAi experiments. To check whether the silencing of these genes influenced the nematode's cold tolerance, a soaking experiment with doublestranded RNA (dsRNA) was set up. The dsRNAs were generated for the selected genes following the instructions of the T7 RiboMAX Express RNAi System (Promega, Madison, Wisconsin, USA) using the primers listed in Table S1. dsRNA of the gene $g f p$ was used as non-native negative control. RNAi soaking was performed by a method modified from Wang et al. (2017). Ditylenchus destructor ( $15 \mathrm{mg})$ were soaked in $200 \mu \mathrm{L}$ of target dsRNAs solution (unigene 15596, unigene 15628 and $g f p$ ) at a final concentration of 1.5 $\mu \mathrm{g} / \mu \mathrm{L}$, and then incubated in a rotator shaker $(100 \mathrm{rpm})$ at 25 ${ }^{\circ} \mathrm{C}$ for $12 \mathrm{~h}$. As control treatments, nematodes were soaked in $\mathrm{ddH}_{2} \mathrm{O}$. After soaking, the viability of the nematodes from the four samples was evaluated by counting the number of living and dead nematodes in each sample. This experiment was repeated thrice. Afterwards, the nematodes from each above treatment were washed. The mRNA expression level of the target genes and adaptability of the nematodes to cold stress were evaluated. qPCR was used to evaluate the mRNA expression levels of unigene 15596 and 15628 after RNAi. The capacity of $D$. destructor to adapt to cold stress after si- 
lencing the unigenes 15596 and 15628 was evaluated according to a previously described method (Ma et al., 2013). Briefly, 1000 nematodes suspended in $1 \mathrm{~mL}$ of $5 \%$ glycerol were placed in individual wells of a 24 -well plate. The nematodes in the plates were exposed to $-8{ }^{\circ} \mathrm{C}$ for $4 \mathrm{~d}$. After cold treatments, nematodes were transferred to Petri dishes $(60$ $\mathrm{mm}$ diameter) containing $9 \mathrm{~mL}$ of distilled water and incubated at $25^{\circ} \mathrm{C}$ for $24 \mathrm{~h}$ to allow recovery of the nematodes. Nematode survival was assessed by microscopic observation, and nematodes were considered dead when showing no response after probing with a needle. Untreated nematodes were used as controls in each of the experiments. Six replicates were run for each sample.

\section{Statistical analysis}

Effects of cold or desiccating conditions on nematode mortality were analyzed with SPSS Statistics 16.0. Data from repeated bioassays that were not significantly different were pooled for analysis. Statistical analyses were performed using one-way or two-way ANOVA, followed by Tukey's multiple means comparison procedure. Percentage data were arcsine transformed before analysis. A $P$ value $<$ 0.05 was considered statistically significant.

\section{Results}

\section{Transcriptome sequencing and assembly}

Each run yielded about 4.5 gigabases $(\mathrm{Gb})$ clean paired end reads, and a total of $22.48 \mathrm{~Gb}$ clean data were obtained with cycle Q20 (base quality greater than 20) > 96\% (Table 1). The GC content of the transcriptomes spanned from $42.31 \%$ to $44.05 \%$. Quality reads from each library were de novo assembled separately into 102,517 unigenes, with an average length of 1,076 bp and the N50 statistical value was $1,870 \mathrm{bp}$. The unigenes total length was $110,389,848 \mathrm{bp}$.

\section{Unigene annotation and classification}

Unigene sequences were aligned by BLASTX $\left(\right.$ E-value $\leq 1 \mathrm{e}^{-5}$ ) to $\mathrm{Nr}$, Nt, Swiss-Prot, GO, COG, KEGG and Interpro databases. Protein function information was predicted from annotation of the most similar protein in these databases. A total of 58,453 unigenes were annotated with known biological functions, comprising $57 \%$ of all the unigenes in the transcriptome libraries. In total, 55,320 (54\%) unigenes were annotated against $\mathrm{Nr}$ database. For the best-

Table 1 - Statistics of reads in the Ditylenchus destructor transcriptomes.

\begin{tabular}{lccccc}
\hline & CK_6 & D_1G & D_2G & D_1W & D_2W \\
\hline Clean reads (Mb) & 29.99 & 29.92 & 29.85 & 30.05 & 29.97 \\
Total clean bases (Gb) & 4.50 & 4.49 & 4.48 & 4.51 & 4.50 \\
Clean reads ratio (\%) & 91.82 & 91.60 & 91.38 & 92.01 & 91.75 \\
Clean reads Q20 (\%) & 96.22 & 96.42 & 96.59 & 96.31 & 96.44 \\
Number of transcripts & 88480 & 86694 & 90581 & 76250 & 76643 \\
Number of unigenes & 60018 & 57932 & 62469 & 51729 & 51466 \\
Unigenes GC contents (\%) & 42.58 & 42.59 & 42.31 & 43.43 & 44.05 \\
\hline
\end{tabular}

matched species distribution, $22.3 \%$ of the distinct sequences mapped to the sequences of Ascaris suum, 12.3\% aligned to the sequences of Ancylostoma ceylanicum, 10.8\% to Loa loa, and $8.3 \%$ mapped to Haemonchus contortus. The searches revealed that $12,227(11.9 \%), 46,874$ (45.7\%), 23,796 $(23.2 \%), 44,135$ (43.1\%), 28,349 (27.7\%) and 45,757 $(44.6 \%)$ of the unigenes had similarity in the Nt, Swiss-Prot, $\mathrm{COG}, \mathrm{KEGG}, \mathrm{GO}$ and Interpro databases, respectively. After functional annotation, 55,476 CDS were detected. CDSs of unigenes that had no hit in blast searches were predicted by ESTScan, and 14,536 unigenes obtained significant blast hits.

All the unigenes were functionally characterized into GO categories based on their similarities to known proteins in $\mathrm{Nr}$ database. Sequences were classified into 58 subcategories belonging to three main GO categories, including biological process (24 subcategories), cellular component (17 subcategories), and molecular function (17 subcategories) (Figure S1A). Within the biological process category, single-organism process and cellular process were predominant. In the category of cellular components, 'cell part' and 'cell' were dominant over the other subgroups. Binding and catalytic activity were the most representative subcategories in the molecular function category. Additionally, pathways were predicted from the KEGG database, and in total, 44,135 unigenes were grouped into 42 pathways (Figure $\mathrm{S} 1 \mathrm{~B})$. The most dominant clusters were related to signal transduction.

\section{SSR and SNP detection}

Simple sequence repeats have commonly been used in genetic diversity, molecular assisted selection, population structure, and genetic mapping studies. SSRs in genes that occur in the protein-coding regions of annotated unigenes could be used to analyze the attributes of functional genes in association with their phenotypes (Zalapa et al., 2012). The transcriptome data was used to analyze the SSRs, because no information is available for SSRs in $D$. destructor. A total of 1154 SSRs distributed over 1078 unigenes were detected. Tri-nucleotides were the most frequent motif types, followed by di-nucleotide and mono-nucleotide. Among the microsatellites detected, the dominant classes of repeating sequences in the unigenes were AT/AT (154) representing $53.5 \%$ of the di-nucleotide SSRs, followed by AAT/ATT (123) representing 25.8\% and AAG/CTT (97) representing $20.3 \%$ of the tri-nucleotide SSRs (Figure 1A). A complete list of SSRs is provided in Table S2.

Due to their wide distributions and abundant polymorphisms, SNPs are useful markers for investigating population genetic structures, the mapping important traits, and for whole-genome association studies (Luo et al., 2016). Functional SNP markers could provide genotype information as they occur in coding regions that may cause a loss or change of protein function (Ellegren, 2008). Each transcriptome generated nearly 60,000 SNPs (Figure 1B). Transitions were more abundant than transversions. The numbers of the transition type $\mathrm{A} / \mathrm{G}$ and $\mathrm{C} / \mathrm{T}$ were equally abundant, and the 


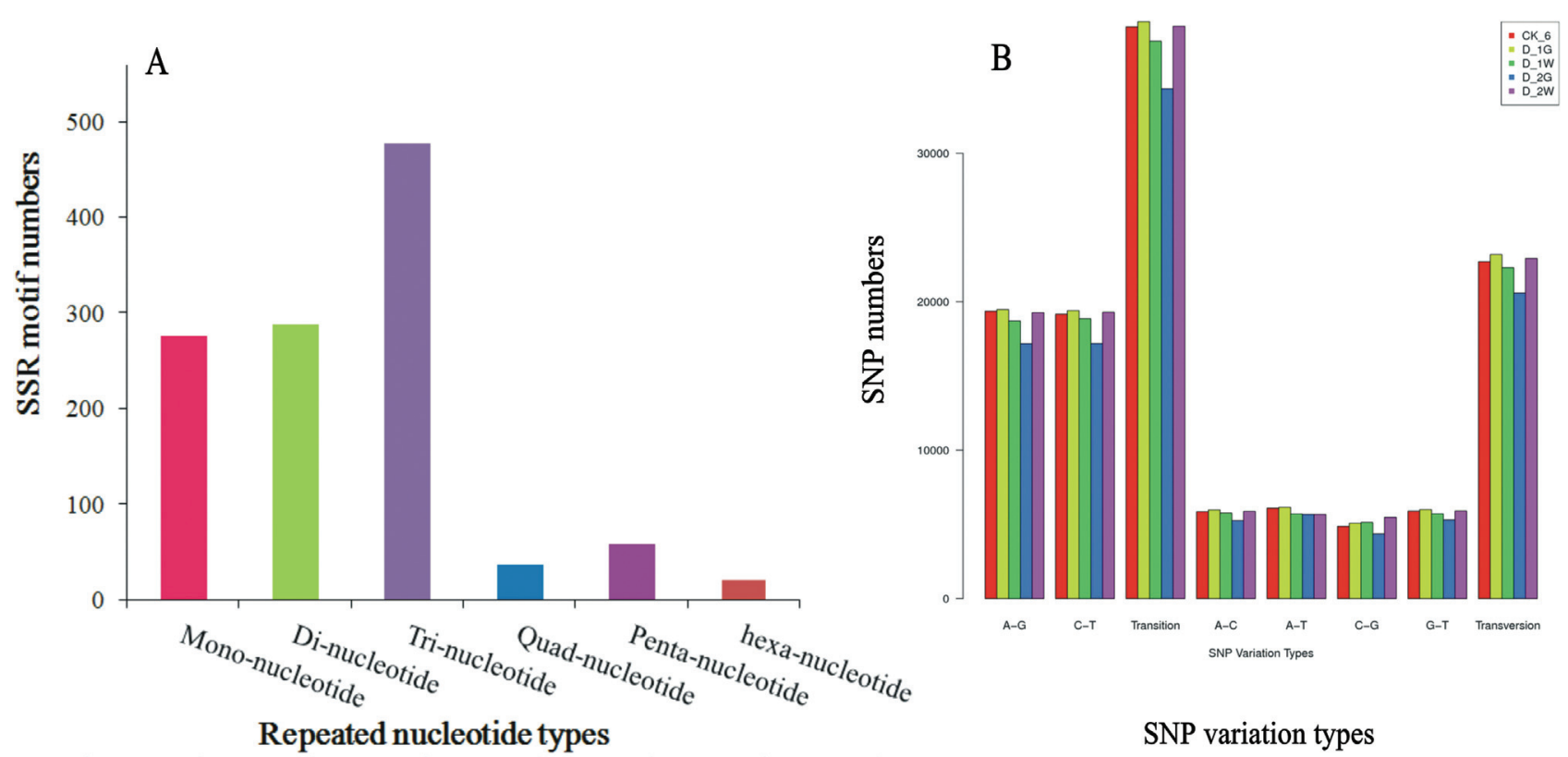

Figure 1 - Simple sequence repeats (SSRs) size distributions (A) and Single nucleotide polymorphisms (SNPs) variant type distributions (B). X axis represents the type of SSRs/ SNP, Y axis represents the number of SSRs/ SNPs.

numbers of the transversion types $\mathrm{A} / \mathrm{T}, \mathrm{A} / \mathrm{C}$, and $\mathrm{G} / \mathrm{T}$ were in the similar level. The statistics of the SNPs validated in all samples are in Table S3.

\section{Changes in gene expression profile among the different nematode samples}

Genes that were highly expressed in both cold-treated samples were considered as cold related genes, and genes that were highly expressed in both desiccation-treated samples were considered as desiccation-related (FDR $\leq 0.001$ and $\log _{2}$ Ratio $\geq 2$ ). A Venn diagram displays the number of unique and shared DEGs amongst the five nematode samples, 41,083 unigenes were shared across all five libraries (Figure 2). The analysis of differentially expressed genes in response to cold revealed 4743 common unigenes in D $1 \mathrm{~W}$, and D_2W, 9303 were specific expressed in D_1W and 9,733 unigenes were specific expressed in D $2 \mathrm{~W} .43 \%$ of these shared unigenes have no annotation. Compared with the control, nematodes in cold conditions had notably higher expression levels for unigenes related to mucin $5 \mathrm{~B}$, serine/threonine protein phosphatase, antifreeze protein, histone acetyl transferase, RAB11 family interacting protein, neuropeptide-like protein 29 (Nlp-29), udp-glucuronosyltransferase and trichohyalin-like (Table S4). Compared with the control nematodes (CK_6), 10,695 and 12,514 unigenes were specifically observed in D_1G and D_2G respectively. Furthermore, 4,587 unigenes were shared across the two desiccation treated samples (Figure 2). For $49 \%$ of these shared unigenes no annotation was found. The highly expressed annotated unigenes included those encoding mucin-5B, GMP synthase, dihydrodiol dehydrogenase, CBR-NURF-1 protein, ubiquitin $\mathrm{C}$ and lipid phosphate phosphohydrolase (Table S4).
Differentially expressed unigenes between pairwise combinations of different nematode samples were identified (Figure S2). In nematodes exposed to cold or desiccation conditions, a number of genes were induced during the nematode acclimation process, and expression of many genes was shut down during the adaption process (Table 2). Compared to the control, nematodes in the cold conditions showed a significantly higher number of down-regulated DEGs than the nematodes in dry conditions, suggesting that more genes were affected by the cold stress. A hierarchical cluster analysis was used to determine the profiles of the DEGs from pairwise comparisons among the control nematodes and the treated nematodes (Figure 3). Unigenes with similar expression patterns were grouped in the same cluster. The analysis showed that the unigenes' expression pattern in the D $1 \mathrm{~W}$ treatment was similar to that of the D $2 \mathrm{~W}$, and the unigenes' expression pattern of D_1G was similar to that of the D_2G.

\section{Functional annotation of differentially expressed unigenes}

We compared the differences occurring between the control and all treated nematodes (Figure S3). With regard to DEGs between the control nematodes and the cold treated

Table 2 - Number of differentially expressed genes (DEGs) in pairwise comparisons between different nematode samples.

\begin{tabular}{lcc}
\hline Samples & Up-regulated & Down-regulated \\
\hline CK_6 vs.D_1G & 5907 & 3911 \\
CK_6 vs.D_2G & 4815 & 5124 \\
CK_6 vs.D_1W & 5125 & 8556 \\
CK_6 vs.D_2W & 5192 & 7846 \\
\hline
\end{tabular}




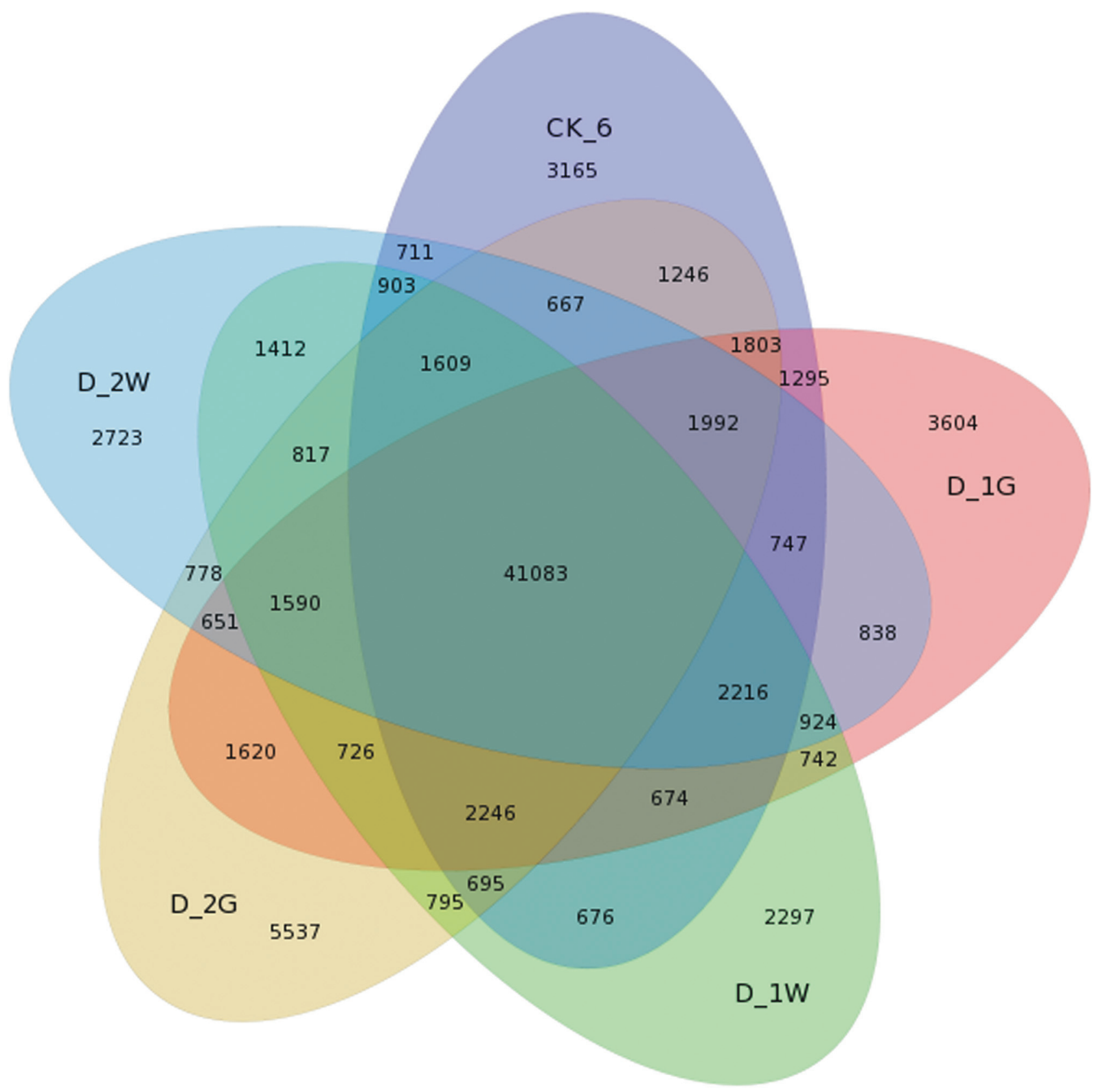

Figure 2 - Venn diagram illustrating common and specifically unigenes in different treatments of Ditylenchus destructor.

nematodes (D_1W and D_2W), the DEGs were significantly enriched $(P<0.05)$ in terms such as sugar-phosphatase activity, cation channel activity, and voltage-gated channel activity. Specifically, GO enrichment showed that the terms of pre-mRNA 3'-splice site binding, voltage-gated ion channel activity, and voltage-gated channel activity were only significantly enriched in cold conditions. Phosphofructokinase activity was significantly enriched in desiccation conditions. Furthermore, GO terms serine-type carboxypeptidase activity, serine-type exopeptidase activity, and alpha-1,3-mannosylglycoprotein 2-beta- $\mathrm{N}$-acetylglucosaminyltransferase activity were significantly up-regulated in the cold treated nematodes (D_1W and D_2W). The GO terms stearoyl-CoA 9-desaturase activity, 6-phosphofructo-2-kinase activity, and fructose-2,6-bisphosphate 2-phosphatase activity showed significant up-regulation in D_1G, and aryl sulfotransferase activity, structural constituent of ribosome, and structural molecule activity were significantly enriched in up-regulated genes of D_2G (Table S5).

All DEGs were mapped to the KEGG database and compared with the whole transcriptome data. Notably, some pathways were only influenced under certain stresses. For instance 9,656 differentially regulated unigenes with KEGG annotation were identified between the CK_6 and D_1W samples, with specific significantly enrichment of genes $(P \leq$ 0.05 ) observed for pathways of nitrogen metabolism, sele- 


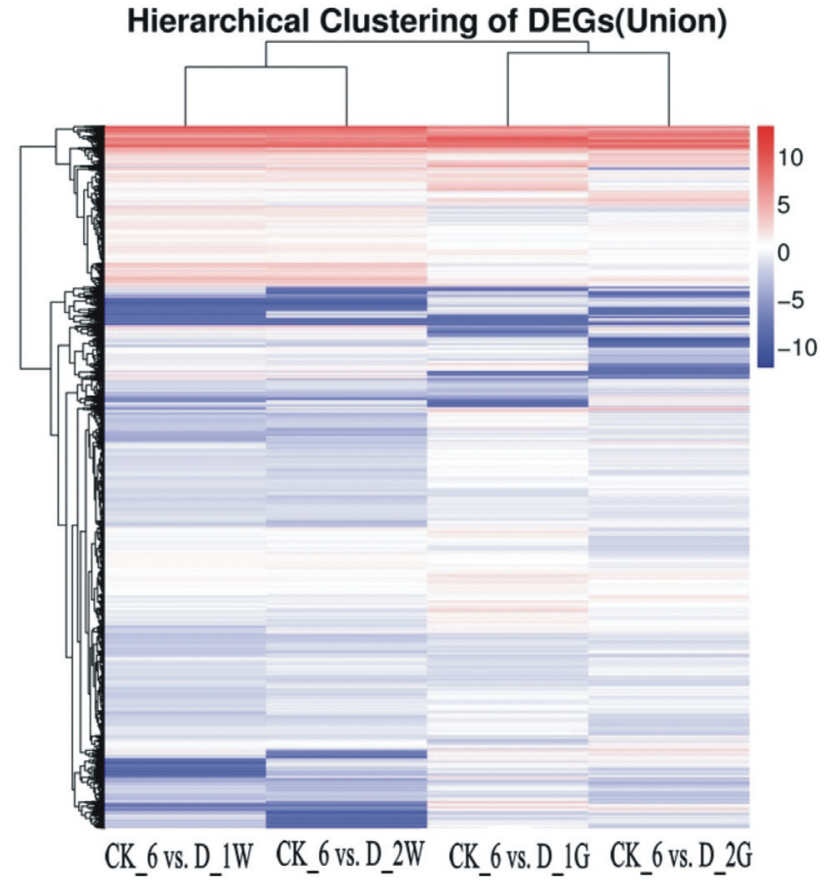

Figure 3 - Heatmap of hierarchical clustering of differentially expressed genes (DEGs). X axis represents each comparing samples. Y axis represents DEGs. Coloring indicates fold change.

no-compound metabolism, and PI3K-Akt signaling pathway. Furthermore, 9,471 DEGs were found when comparing CK_6 and D_2W, with specific significantly enrichment of hypertrophic cardiomyopathy, other glycan degradation, biotin metabolism, and the serotonergic synapse pathways. In D_1W and D_2W, the pathways of retinol metabolism, spliceosome, and pentose and glucuronate interconversions were significantly up-regulated; neuroactive ligand-receptor interaction, calcium signaling pathway, fructose and mannose metabolism, and lipoic acid metabolism were significantly down-regulated (Table S6). Another 6,878 differentially regulated unigenes were identified between the CK_6 and D_1G libraries. Among these, genes associated with glycine-serine and threonine metabolism, biosynthesis of unsaturated fatty acids, arachidonic acid metabolism, linoleic acid metabolism, carbon metabolism, and fatty acid metabolism were functionally specific significantly enriched. A further 7,169 differentially regulated unigenes were identified between the CK_6 and D_2G libraries, with specific significantly enrichment of unigenes for pathways involved in 2-oxocarboxylic acid metabolism, degradation of aromatic compounds, antigen processing and presentation, lysine degradation, fat digestion and absorption, and pyrimidine metabolism. Pathways of chemical carcinogenesis, metabolism of xenobiotics by cytochrome P450, steroid hormone biosynthesis, ascorbate and aldarate metabolism, ether lipid metabolism and PPAR signaling pathway were enriched among the up-regulated genes of D_1G and D_2G; in contrast, long-term potentiation was significantly down-regulated in both D_1G and D_2G (Table S6). The up-regulated pathways of pentose and glucuronate interconversions, retinol metabolism, were commonly shared by all the four treated samples. These results indicate that DEGs involved in these pathways may play an important role in the adaption of harsh environmental conditions.

\section{Validation of DEGs data by Quantitative RT-PCR}

No amplification was found in no-template controls, and single gene specific melting curves confirmed the specificity of the qRT-PCR assays. Fold changes from qPCR assays were compared with the RNA-seq expression profiles (Figure 4, Table S4). In general, all unigenes showed similar expression patterns with the RNA-seq data, adding confidence to the RNA-seq data analysis.

\section{The effect of RNAi}

Compared with the control treatments, the relative expression levels of the unigenes 15628 and 15596 were significantly reduced in the dsRNA-treated nematodes $(F=30.78$; $\mathrm{df}=2,6 ; P<0.01 ; F=129.59 ; \mathrm{df}=2,6 ; P<0.01$ ) (Figure $5 \mathrm{~A}$ ), with an average reduction of $30 \%$ for unigene 15628 and $45 \%$ for unigene 15596 in the dsRNA soaked nematodes compared to the control group. dsRNA had no effect on the survival rate of $D$. destructor, and nematode survival among different treatments did not statistically differ and remained above $95 \%$ before exposure to $-8{ }^{\circ} \mathrm{C}$. The effect of silencing the unigenes 15628 and 15596 on cold tolerance was studied (Figure 5B). Nematodes were transferred into 5\% glycerol before being exposed to $-8{ }^{\circ} \mathrm{C}$. Glycerol was used as plasma membrane-permeant cryoprotectant to inhibit intracellular ice formation (Storey et al., 1998). The unigenes 15628 and 15596 dsRNA-treated nematodes displayed significantly higher mortality compared to the $g f p$ dsRNA-treated and none-treated nematodes after subsequent exposure to $-8{ }^{\circ} \mathrm{C}$ $(F=37.57 ; \mathrm{df}=2,15 ; P<0.01 ; F=18.36 ; \mathrm{df}=2,15 ; P<$ $0.01)$. Knockdown of each gene led to decreased cold tolerance of $D$. destructor.

\section{Discussion}

The aim of the present study was to characterize and study the genes related to cold and dessication tolerance of D. destructor. Differential gene expression levels across control and the treated nematode samples were analyzed. In response to the stress, the nematodes activated metabolic pathways shortly after exposure to different stresses. Not surprisingly, some reported stress-related genes were in some way involved in the stress response, such as genes encoding the LEA and HSP proteins. LEA proteins play an important role during desiccation in nematodes (Adhikari et al., 2010; Erkut et al., 2013). We recorded 14 unigenes related to LEAs in D. destructor using BLASTX similarity searches of our transcriptome sequences against the $\mathrm{Nr}$ databases, with nine LEA related genes being up-regulated during desiccation and also cold stress of D. destructor (Table S4). The HSP families contain various genes that could be either induced by stress treatment or constitutively expressed in nematodes (Yaari et al., 2016). Our results showed that the small 


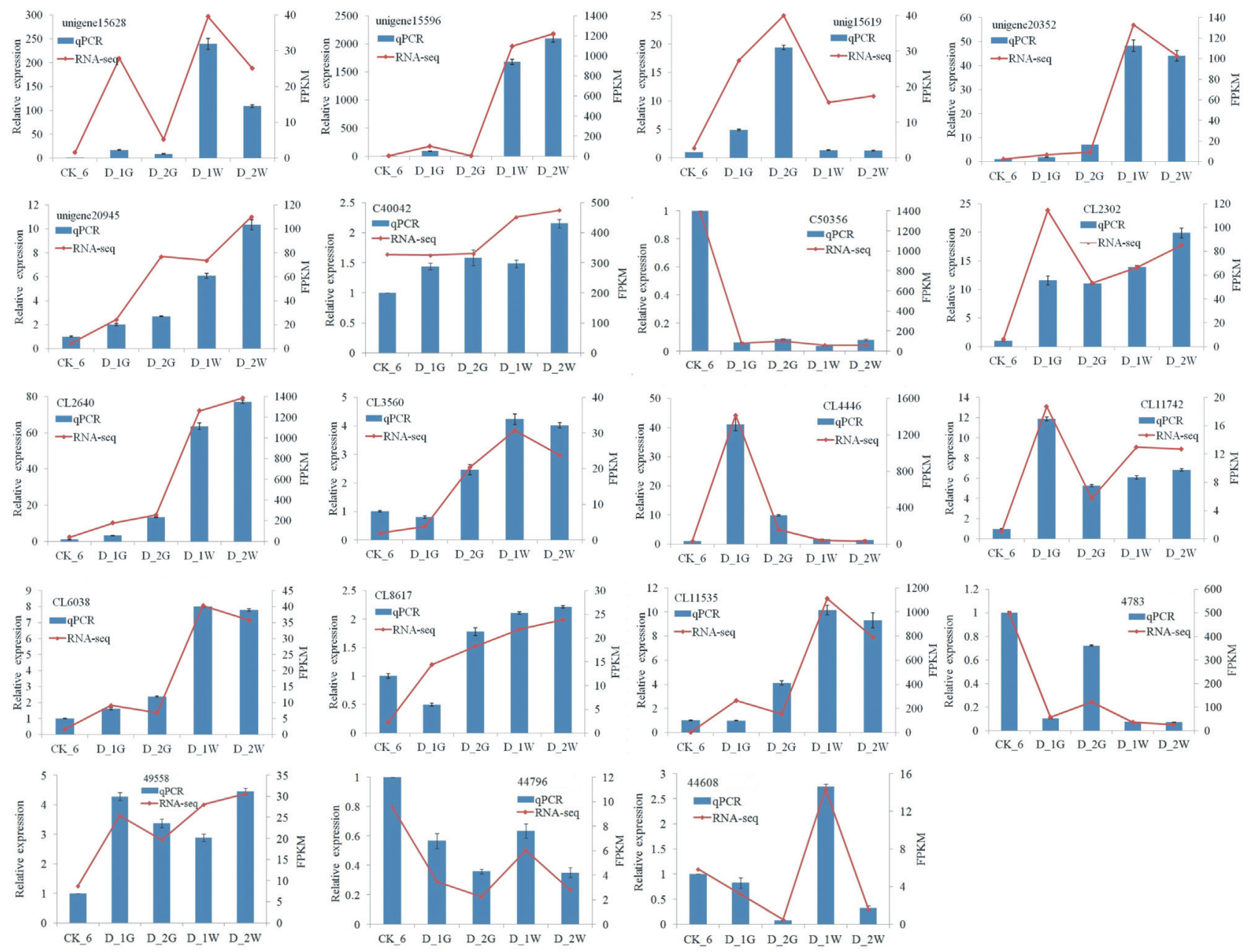

Figure 4 - RNA-seq and RT-qPCR analyses of expression levels of tolerance related genes in different nematode samples. X-axis indicates nematode samples, and y-axis indicates gene expression levels by qPCR (left) and FPKM (right). Histograms indicate the relative expression value obtained by qPCR; the solid lines in all plots indicate the FPKM values obtained by RNA-seq.

HSP21-like and small HSP12.6 proteins were highly expressed following cold stress in D. destructor. HSP20 and HSP70-A were highly up-regulated during exposure to desiccation, whereas other heat shock proteins in $D$. destructor were constitutively expressed or slightly up or downregulated. The induction or inhibition of various heat shock proteins might be necessary for $D$. destructor to adapt to cold or desiccation conditions.

The TPS and aquaporin proteins were reported to enhance desiccation resistance in many nematodes (Gal et al., 2005; Adhikari et al., 2010; Thorne et al., 2014). Two TPS genes were abundantly expressed in both cold and desiccation treated $D$. destructor. However, exposure to desiccation and cold did not lead to marked changes in the expression levels of aquaporin genes in $D$. destructor.

Some genes that did not show any relationship with nematode temperature or dessication tolerance in former reports were found significantly higher expressed in the cold or dessication treated $D$. destructor nematodes, such as genes encoding NLP-29 protein, mucins, UDP-glucuronosyltransferases (UGTs). The NLP-29 protein plays a role in response to physical injury and osmotic stress in C. elegans (Pujol et al., 2008a,b). In D. destructor, NLP-29 was up-regulated in response to cold stress, suggesting that NLP-29 may be involved in the cold survival of the nematodes. Mucins have a direct role in combating pathogens and parasites in mice and are considered as important parts in the coordinated immune response (Hasnain et al., 2013). Parsons et al. (2014) reported that mucin protects C. elegans against gram negative bacteria. Our study suggested that mucins may not only protect nematodes from biotic stressors, but may also provide protection against abiotic stressors, such as cold and desiccation. Certainly, further evidence is required to support the role for mucins in $D$. destructor.

The serine/threonine phosphatases proteins (STPs) have critical functions in the regulation of adaptive stress responses in plants, such as salt, drought, and cold stress (País et al., 2009; Cui et al., 2013). In animals, STPs play extremely diverse functions, such as sperm motility, cellular survival, and development and stress response ( $\mathrm{Lu}$ et al., 2007; Kolupaeva, 2019). In nematodes, such as C. elegans, Trichostrongylus colubriformis and T. vitrinus, STPs are in- 

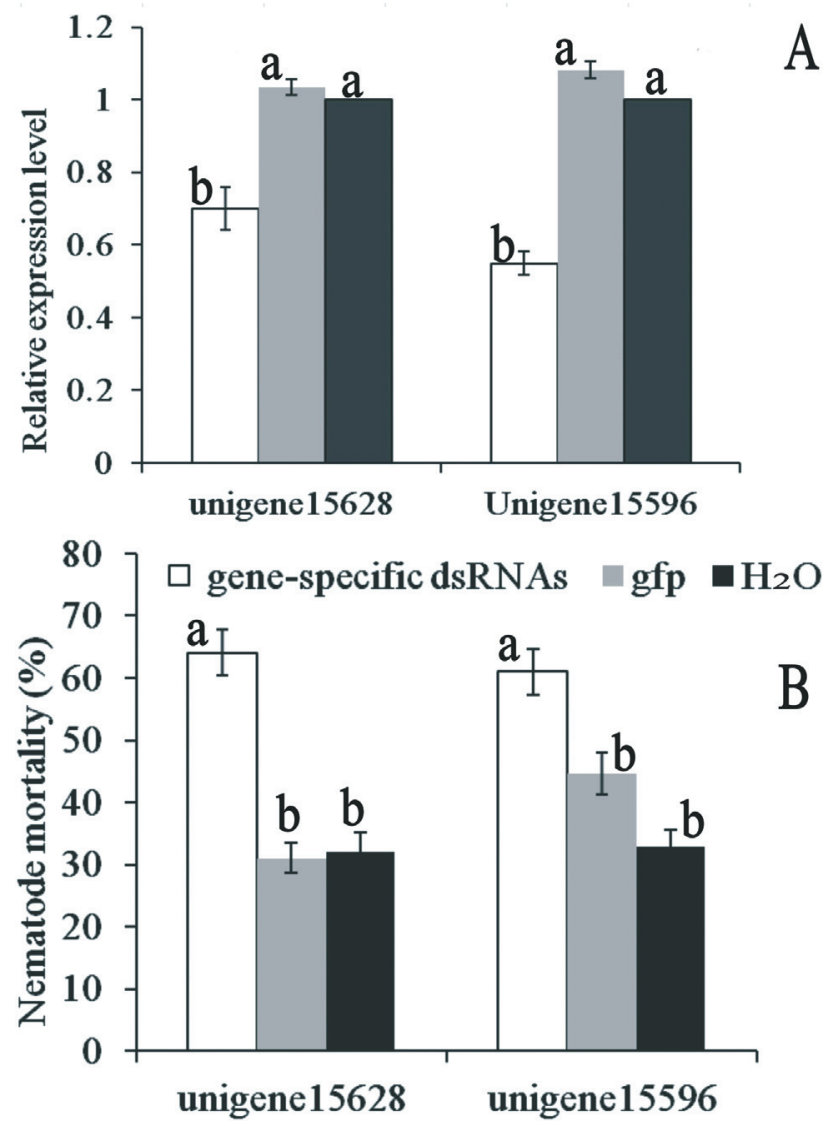

Figure 5 - Effect of RNAi on cold tolerance of Ditylenchus destructor. (A) Gene expression analysis by qPCR of unigene 15628 and 15596 after RNAi. Nematodes were incubated in unigene 15628 and 15596 dsRNA for $24 \mathrm{~h}$. Control nematodes were incubated in $\mathrm{dd}_{2} \mathrm{O}$ without dsRNA. (B) Mean mortality $( \pm \mathrm{SE})$ of control and dsRNA-treated nematodes after exposure to $-8{ }^{\circ} \mathrm{C}$ for 4 days. Silencing unigene 15628 and 15596 promoted a significant increase in mortality $(64 \% \pm 3.6 \%, 61 \% \pm 3.7 \%)$ when compared to control nematodes $(32 \% \pm 2.9 \%, 33 \% \pm 2.8 \%)$. Bars with the same letter(s) do not differ significantly according to Tukey's test $(P>$ $0.05)$

volved in signal transduction or transcriptional activation and play key roles in development, reproductive, and survival (Barford et al., 1998; Cantacessi et al., 2010; Campbell et al., 2011). This study showed that unigenes encoding STPs are up-regulated during cold stress of $D$. destructor. It is possible that STPs play a role in the cold survival of nematodes, as they are thought to do in plants.

UDP-glucose:glycoprotein glucosyltransferase (UGT) are enzymes of a detoxification system and are important for the maintenance of organism health (Laing et al., 2010). The expression of UDP-glucose:glycoprotein glucosyltransferase was induced by temperature stress in Schizosaccharomyces pombe (Fernandez et al., 1996). The induction of UGTs unigenes by cold and desiccation in $D$. destructor suggest that these unigenes may be stress related. Desiccation stress induction of ubiquitin genes occurs in Antarctic nematode (Adhikari et al., 2009). The ubiquitin C-terminal hydrolase was reported to maintain osmotic balance and execute actin-dependent processes in the early $C$. elegans embryo
(Kaitna et al., 2002). Consistent with these observations, unigenes encoding ubiquitin-related proteins were up-regulated during desiccation stress of $D$. destructor. Cold and desiccation tolerance may elicit similar responses to injury during periods of extreme environmental conditions (Adhikari and Adams, 2011). In D. destructor, some genes were highly expressed in response to both of cold and desiccation stress, such as genes encoding proteins involved in mucin $5 \mathrm{~B}$, gut esterase, and protein DHS-7. By contrast, some genes were only activated during cold or desiccation in $D$. destructor. There was also a large number of unigenes that were down-regulated in the stress treated nematodes, such as genes encoding protein HSP-12.2, aldolase, nose resistant to fluoxetine protein 6 (NRF6). Expression levels of aldolase were reduced in response to heat stress in maize (Duke and Doehlert, 1996). The NRF protein is associated with drug and yolk transport across the basal intestinal membrane surface in C. elegans (Choy et al., 2005). Our results show that $D$. destructor actively responds to stress via expression of a series of genes include the above genes. However, how these up/down-regulated genes regulate cold and desiccation tolerance in $D$. destructor requires further studies.

Ditylenchus destructor survives low temperature and desiccation for a long period without food (Lin et al., 1997). Nematodes respond to stress in a complex fashion, with many transcriptional changes that include up- or downregulated DEGs or pathways. Functional annotations of DEGs make it possible to know the molecular mechanisms underlying specific biological processes of different treated nematodes. However, some of the unigenes had no known homolog in any nematode or any other existing organisms. New genes or molecular markers related to different stages formation might be identified from this group in the future. We compared the cold-treated and desiccation treated groups to the untreated nematodes and found some similar responses. Further investigations, for example, correlation between desiccation and freeze tolerance, will be assessed in further studies.

Differentially expressed genes that include up-regulated and down-regulated genes were subjected to qRT-PCR analysis to validate the accuracy and reproducibility of the Illumina RNA-seq results. The selected genes encoding heat shock proteins, antifreeze proteins, and CRE-FAT-6 were reported as nematode stress-related genes. The expression results of RNA-Seq and qRT-PCR showed good consistency for all unigenes, which increases confidence in the RNAseq. In each sample, billions of nematodes were pooled for RNA-seq. Different expressed genes were obtained by comparing of different groups. The results of the group change tendency are representative.

Knockdown of the unigenes 15628 and 15596 led to a decreased ability of the nematode's cold tolerance. While, RNAi of the unigenes 15628 and 15596 in D. destructor produced no difference in nematode survival with respect to controls before exposure to cold conditions, mortality increased in the treated nematodes after exposure. The uni- 
genes 15628 and 15596 were annotated as gut esterase 1 (ges-1) and antifreezing protein, respectively.

The gut-specific esterase gene (ges-1) is normally expressed in the intestine of C. elegans (Kennedy et al., 1993). C. elegans ges- 1 gene expression is dependent on WGATAR sites, and deletion of a tandem pair of WGATAR sites abolishes ges- 1 expression in the gut, but simultaneously activates ges- 1 expression in both the anterior and posterior pharynx, as well as in the rectum (Fukushige et al., 2005). ges-1 expression is responsible for approximately half of the total esterase activity in worms (McGhee et al., 1990) and likely has important roles in the metabolism of exogenous molecules and nutrients (Egan et al., 1995). Burns et al. (2017) reported that GES-1 hydrolyzes the nematicide wact-86, which is lethal to C. elegans. Marshall and McGhee (2001) suggest that pharyngeal expression of ges- 1 is advantageous only under certain developmental or environmental conditions in C. briggsae.

Antifreeze proteins assist nematode cold tolerance by freeze avoidance and cryoprotective dehydration (Wharton, 2011). Adhikari et al. (2010) reported that the afp gene was significantly up-regulated during exposure and recovery from freezing in $P$. murrayi. Our studies showed the important role of the unigenes 15628 and 15596 in response to cold conditions. However, there are open question. How is genes expression coordinated between the different components of the organ system? Is expression of these genes modulated by other transcription factors? What are the details for the physiological functions of the antifreeze proteins in $D$. destructor? Future investigation is required to unravel the functions of these genes.

This study provides the first insights into the cold and desiccation induced transcriptome of $D$. destructor. We did not use genomic data (Zheng et al., 2016) because of insufficiency in assembly level of 'Contig' and annotation information. The de novo transcriptome assembly could provide a reliably results (Grabherr et al., 2011; Haas et al., 2013). We investigated the molecular characteristics of cold and desiccation treated nematodes and showed that the tolerance process involves complex traits. These findings provide a substantial contribution to existing sequence resources of $D$. destructor, and the annotated transcriptome sequences and gene expression profiles of $D$. destructor will contribute to explorations of the molecular mechanisms of environmental adaptation and the development of new effective control strategies.

\section{Acknowledgments}

We appreciate Beijing Genomics Institute at Shenzhen, China for help in sequencing. The authors thank Prof. David Stanley for the advice on English writing. This work was financially supported by the National Natural Science Foundation of China (31401721, 31501616), China Agriculture Research System (CARS-10-B16) and HAAFS Agriculture Science and Technology Innovation Project.

\section{Conflict of Interest}

The authors declare that they have no conflict of interest.

\section{Author Contributions}

JM, BG performed the experiments. JM, SLC analyzed the data. BG, XHL and RYW contributed reagents/materials/analysis tools. JM and SLC wrote the paper. All authors read and approved the final version.

\section{References}

Adhikari BN and Adams BJ (2011) Molecular analysis of desiccation survival in Antarctic nematodes. In: Perry RN and Wharton DA (eds) Molecular and physiological basis of nematode survival. CABI Press, Wallingford, pp 205-232.

Adhikari BN, Wall DH and Adams BJ (2009) Desiccation survival in an Antarctic nematode: Molecular analysis using expressed sequenced tags. BMC Genomics 10:69.

Adhikari BN, Wall DH and Adams BJ (2010) Effect of slow desiccation and freezing on gene transcription and stress survival of an antarctic nematode. J Exp Biol 213:1803-1812.

Ali F and Wharton DA (2016) Ice-active substances from the infective juveniles of the freeze tolerant entomopathogenic nematode, Steinernema feltiae. PLoS One 11:e0156502.

Barford D, Das AK and Egloff MP (1998) The structure and mechanism of protein phosphatases: Insights into catalysis and regulation. Annu Rev Biophys Biomol Struct 27:133-164.

Benjamini Y and Yekutieli D (2001) The control of the false discovery rate in multiple testing under dependency. Ann Stat 29:1165-1188.

Burns AR, Bagg R, Yeo M, Luciani GM, Schertzberg M, Fraser AG and Roy PJ (2017) The novel nematicide wact-86 interacts with aldicarb to kill nematodes. PLoS Neglected Trop Dis 11:e0005502.

Campbell BE, Hofmann A, McCluskey A and Gasser RB (2011) Serine/threonine phosphatases in socioeconomically important parasitic nematodes - prospects as novel drug targets? Biotechnol Adv 29:28-39.

Cantacessi C, Mitreva M, Campbell BE, Hall RS, Young ND, Jex AR, Ranganathan S and Gasser RB (2010) First transcriptomic analysis of the economically important parasitic nematode, Trichostrongylus colubriformis, using a next-generation sequencing approach. Infect Genet Evol 10:1199-1207.

Choy RK, Kemner JM and Thomas JH (2005) Fluoxetine-resistance genes in Caenorhabditis elegans function in the intestine and may act in drug transport. Genetics 172:885-892.

Chen Q, Li D, Wang F, Zhang R and Ling Y (2017) Trehalose metabolism genes of Aphelenchoides besseyi (Nematoda: Aphelenchoididae) in hypertonic osmotic pressure survival. Open Biol 6:664-672.

Conesa A, Gotz S, Garcia-Gomez JM, Terol J, Talon M and Robles M (2005) Blast2GO: A universal tool for annotation, visualization and analysis in functional genomics research. Bioinformatics 21:3674-3676.

Cui MH, Yoo KS, Hyoung S, Nguyen HTK, Kim YY, Kim HJ, Ok SH, Yoo SD and Shin JS (2013) An Arabidopsis R2R3-MYB transcription factor, AtMYB20, negatively regulates type $2 \mathrm{C}$ serine/threonine protein phosphatases to enhance salt tolerance. FEBS Lett 587:1773-1778. 
Duke ER and Doehlert DC (1996) Effects of heat stress on enzyme activities and transcript levels in developing maize kernels grown in culture. Environ Exp Bot 36:199-208.

Egan CR, Chung M, Allen FL, Heschl MF, Van Buskirk CL and McGhee JD (1995) A gut-to-pharynx/tail switch in embryonic expression of the Caenorhabditis elegans ges-1 gene centers on two GATA sequences. Dev Biol 170:397-419.

Ellegren H (2008) Comparative genomics and the study of evolution by natural selection. Mol Ecol 17:4586-4596.

Erkut C, Vasilj A, Boland S, Habermann B, Shevchenko A and Kurzchalia TV (2013) Molecular strategies of the Caenorhabditis elegans dauer larva to survive extreme desiccation. PLoS ONE 8:e82473.

Fernandez F, Jannatipour M, Hellman U, Rokeach LA and Parodi AJ (1996) A new stress protein: synthesis of Schizosaccharomyces pombe UDP-Glc: Glycoprotein glucosyltransferase mRNA is induced by stress conditions but the enzyme is not essential for cell viability. EMBO J 15:705-713.

Fukushige T, Goszczynski B, Yan J and McGhee JD (2005) Transcriptional control and patterning of the pho-1 gene, an essential acid phosphatase expressed in the $C$. elegans intestine. Dev Bio 279:446-461.

Gal T, Solomon A, Glazer I and Koltai H (2001) Alteration in the levels of glycogen and glycogen synthase transcripts during desiccation in the insect-killing, highly desiccation tolerant nematode Steinernema feltiae IS-6. J Parasitol 87:725-732.

Gal T, Glazer I and Koltai H (2004) A LEA3 family member is involved in survival of $C$. elegans during exposure to dehydration stress. FEBS Lett 577:21-26.

Gal T, Glazer I, Sherman A and Koltai H (2005) Protein interaction of Nucleosome assembly Protein-1 and casein kinase 2 during desiccation response in the insect-killing nematode Steinernema feltiae IS-6. J Parasitol 91:691-693.

Grabherr MG, Haas BJ, Yassour M, Levin JZ, Thompson DA, Amit I, Adiconis X, Fan L, Raychowdhury R, Zeng Q et al. (2011) Full-length transcriptome assembly from RNA-Seq data without a reference genome. Nat Biotechnol 29:644-652.

Goyal K, Walton LJ, Browne JA, Burnell AM and Tunnacliffe A (2005) Molecular anhydrobiology: Identifying molecules implicated in invertebrate anhydrobiosis. Integr Comp Biol 45:702-709.

Haas BJ, Papanicolaou A, Yassour M, Grabherr M, Blood PD, Bowden J, Couger MB, Eccles D, Li B, Lieber M et al. (2013) De novo transcript sequence reconstruction from RNA-seq using the Trinity platform for reference generation and analysis. Nat Protoc 8:1494-1512.

Hasnain SZ, Gallagher AL, Grencis RK and Thornton DJ (2013) New role for mucins in immunity: Insights from gastrointestinal nematode infection. Int J Biochem Cell B 45:364-374.

Iseli C, Jongeneel CV and Bucher P (1999) ESTScan: A program for detecting, evaluating, and reconstructing potential coding regions in EST sequences. Proc Int Conf Intell Syst Mol Biol 99:138-148.

Kaitna S, Schnabel H, Schnabel R, Hyman AA and Glotzer M (2002) A ubiquitin C-terminal hydrolase is required to maintain osmotic balance and execute actin-dependent processes in the early C. elegans embryo. J Cell Sci 115:2293-2302.

Kennedy BP, Aamodt EJ, Allen FL, Chung MA, Heschl MF and McGhee JD (1993) The gut esterase gene (ges-1) from the nematodes Caenmorhabditis elegans and Caenorhabditis briggsae. J Mol Biol 229:890-908.

Kim D, Langmead B and Salzberg SL (2015) HISAT: A fast spliced aligner with low memory requirements. Nat Methods 12:357-360.
Kolupaeva V (2019) Serine-threonine protein phosphatases: Lost in translation. Biochim Biophys Acta Mol Cell Res 1866:83-89.

Laing ST, Ivens A, Laing R, Ravikumar S, Butler V, Woods DJ and Gilleard JS (2010) Characterization of the xenobiotic response of Caenorhabditis elegans to the anthelmintic drug albendazole and the identification of novel drug glucoside metabolites. Biochem J 432:505-516.

Langmead B and Salzberg SL (2012) Fast gapped-read alignment with Bowtie 2. Nat Methods 9:357-359.

Li B and Dewey C (2011) RSEM: Accurate transcript quantification from RNA-Seq data with or without a reference genome. BMC Bioinformatics 12:323-339.

Lin MS, Liu XY, Wen L and Fang ZD (1997) Observations on the overwintering of Ditylenchus destructor in sweet potato and its tolerance against $\mathrm{pH}$ and salt concentration. Jiangsu J Agric Sci 13:36-39.

Lu G, Ren S, Korge P, Choi J, Dong Y, Weiss J, Koehler C, Chen J and Wang Y (2007) A novel mitochondrial matrix serine/threonine protein phosphatase regulates the mitochondria permeability transition pore and is essential for cellular survival and development. Genes Dev 21:784-796.

Luo H, Xiao S, Ye H, Zhang Z, Lv C, Zheng S, Wang Z and Wang $X$ (2016) Identification of immune-related genes and development of SSR/SNP markers from the spleen transcriptome of Schizothorax prenanti. PLoS One 11:e0152572.

Ma J, Chen S, Moens M, De Clercq P, Li X and Han R (2013) Characterization in biological traits of entomopathogenic nematodes isolated from North China. J Invertebr Pathol 114:268276.

Marshall SD and McGhee JD (2001) Coordination of ges- 1 expression between the Caenorhabditis pharynx and intestine. Dev Biol 239:350-363.

McGhee JD, Birchall JC, Chung MA, Cottrell DA, Edgar LG, Svendsen PC and Ferrari DC (1990) Production of null mutants in the major intestinal esterase gene (ges- 1 ) of the nematode Caenorhabditis elegans. Genetics 125:505-514.

McKenna A, Hanna M, Banks E, Sivachenko A, Cibulskis K, Kernytsky A, Garimella K, Altshuler D, Gabriel S, Daly M et al. (2010) The Genome Analysis Toolkit: A MapReduce framework for analyzing next-generation DNA sequencing data. Genome Res 20:1297-1303.

Mortazavi A, Williams BA, McCue K, Schaeffer L and Wold B (2008) Mapping and quantifying mammalian transcriptomes by RNA-Seq. Nat Methods 5:621-628.

Ohta A, Ujisawa T, Sonoda S and Kuhara A (2014) Light and pheromone-sensing neurons regulates cold habituation through insulin signalling in Caenorhabditis elegans. Nat Commun 5:4412.

Parsons LM, Mizanur RM, Jankowska E, Hodgkin J, O'Rourke D, Stroud D, Ghosh S and Cipollo JF (2014) Caenorhabditis elegans bacterial pathogen resistant bus-4 mutants produce altered mucins. PLoS One 9:e107250.

País SM, Téllez-Iñón MT and Capiati DA (2009) Serine/threonine protein phosphatases type $2 \mathrm{~A}$ and their roles in stress signaling. Plant Signal Behav 4:1013-1015.

Pertea G, Huang X, Liang F, Antonescu V, Sultana R, Karamycheva S, Lee Y, White J, Cheung F, Parvizi B et al. (2003) TIGR Gene Indices clustering tools (TGICL): A software system for fast clustering of large EST datasets. Bioinformatics 19:651-652.

Peng H, Gao BL, Kong LA, Yu Q, Huang WK, He XF, Long HB and Peng DL (2013) Exploring the host parasitism of the migratory plant-parasitic nematode Ditylenchus destuctor by expressed sequence tags analysis. PLoS One 8:e69579. 
Pfaffl MW (2001) A new mathematical model for relative quantification in real-time RT-PCR. Nucleic Acids Res 29:e45.

Pujol N, Cypowyj S, Ziegler K, Millet A, Astrain A, Goncharov A, Jin Y, Chisholm AD and Ewbank JJ (2008a) Distinct innate immune responses to infection and wounding in the $C$. elegans epidermis. Curr Biol 18:481-489.

Pujol N, Zugasti O, Wong D, Couillault C, Kurz CL, Schulenburg $\mathrm{H}$ and Ewbank JJ (2008b) Anti-fungal innate immunity in $C$. elegans is enhanced by evolutionary diversification of antimicrobial peptides. PLoS Pathogens 4:e1000105.

Qi Y, Li X, Ma J, Li M and Chen S (2008) The infection and dynamics of Ditylenchus destructor in sweet potato. Acta Agricul Boreali-Sinica 23:234-237.

Quevillon E, Silventoinen V, Pillai S, Harte N, Mulder N, Apweiler $R$ and Lopez R (2005) InterProScan: Protein domains identifier. Nucleic Acids Res 33:116-120.

Ring RA and Danks HV (1998) The role of trehalose in coldhardiness and desiccation. CryoLetters 19:275-282.

Savory FR, Sait SM and Hope IA (2011) DAF-16 and $\Delta^{9}$ desaturase genes promote cold tolerance in long-lived Caenorhabditis elegans age-1 mutants. PLoS One 6:e24550.

Sturhan D and Brzeski MW (1991) Stem and bulb nematodes, Ditylenchus spp. In: Nickle WR (ed) Manual of Agricultural Nematology. Marcel Dekker, New York, pp 423-464.

Storey BT, Noiles EE and Thompson KA (1998) Comparison of glycerol, other polyols, trehalose, and raffinose to provide a defined cryoprotectant medium for mouse sperm cryopreservation. Cryobiology 37:46-58.

vilponis E, Hiiesaar K, Kaart T, Metspalu L, Mänd M, Ploomi A, Williams IH and Luik A (2011) Low temperature survival of post-eclosion stages of the potato rot nematode Ditylenchus destructor Thorne (Tylenchida: Anguinidae). Nematology 13:977-983.

Thorne MAS, Kagoshima H, Clark MS, Marshall CJ and Wharton DA (2014) Molecular analysis of the cold tolerant Antarctic nematode, Panagrolaimus davidi. PLoS One 9:e104526.

Wang BY, Wen RR and Ma L (2017) Molecular characterization and functional analysis of a glutathione peroxidase gene from Aphelenchoides besseyi (Nematoda: Aphelenchoididae). Acta Parasitol 62:565-574.

Wang H, Mao J, Li R, Luo D, Zhao G and Li H (2016) A culture technique for Ditylenchus destructor on sweet potato (Ipomoea batatas). Nematology 18:501-503.

Wang L, Feng Z, Wang X and Zhang X (2010) DEGseq: An R package for identifying differentially expressed genes from RNA-Seq data. Bioinformatics 26:136-138.

Wang RY, Gao B, Ma J, Li XH and Chen SL (2018) Efficacy of different nematicides for controlling Ditylenchus destructor in sweetpotato fields. J Shanxi Agric Univ 38:45-47.

Wharton DA (2011) Cold tolerance. In: Perry RN and Wharton DA (eds) Molecular and physiological basis of nematode survival. CABI Publishing, Wallingford, pp 182-218.

Wharton DA and Raymond MR (2015) Cold tolerance of the Antarctic nematodes Plectus murrayi and Scottnema lindsayae. J Comp Physiology B 185:281-289.
Wharton DA, Barrett J, Goodall G, Marshall CJ and Ramløv H (2005) Ice-active proteins from the Antarctic nematode Panagrolaimus davidi. Cryobiology 51:198-207.

Yaari M, Doron-Faigenboim A, Koltai H, Salame L and Glazer I (2016) Transcriptome analysis of stress tolerance in entomopathogenic nematodes of the genus Steinernema. Int J Parasitol 46:83-95.

Ye J, Fang L, Zheng H, Zhang Y, Chen J, Zhang Z, Wang J, Li S, Li R, Bolund L et al. (2006) WEGO: A web tool for plotting GO annotations. Nucleic Acids Res 34:293-297.

Zalapa JE, Cuevas H, Zhu H, Steffan S, Senalik D, Zeldin E, McCown B, Harbut R and Simon P (2012) Using next-generation sequencing approaches to isolate simple sequence repeat (SSR) loci in the plant sciences. Am J Bot 99:193-208.

Zhang X, Dai P, Gao Y, Gong X, Cui H, Jin Y and Zhang Y (2017) Transcriptome sequencing and analysis of zinc-uptakerelated genes in Trichophyton mentagrophytes. BMC Genomics 18:888.

Zheng J, Peng D, Chen L, Liu H, Chen F, Xu M, Ju S, Ruan F and Sun M (2016) The Ditylenchus destructor genome provides new insights into the evolution of plant parasitic nematodes. Proc Biol Sci 283:20160942.

\section{Internet Resources}

EPPO (2018) European and Mediterranean Plant Protection Organization - EPPO, https://gd.eppo.int/taxon/DITYDE/distribution (accessed October 2018).

FAOSTAT (2016) Food and Agriculture Organization Corporate Statistical Database, http://faostat3.fao.org/browse/Q/QC/E (accessed 13 February 2016).

\section{Supplementary material}

The following online material is available for this article:

Figure S1 - Functional distribution of GO annotation and KEGG annotation.

Figure S2 - Volcano plots representing the differentially expressed genes (up and down-regulated) in different treated samples as compared to the control nematodes.

Figure S3 - Ditylenchus destructor in different conditions.

Table S1 - List of primers used in the study.

Table S2 - List and features of SSRs.

Table S3 - Statistic of SNPs that validated in all samples.

Table S4 - Genes highly expressed in both cold and desiccation treated nematodes.

Table S5 - Significantly enriched GO terms in the treated samples.

Table S6 - Significantly enriched KEGG pathways in the treated samples.

Associate Editor: Guilherme Corrêa de Oliveira

License information: This is an open-access article distributed under the terms of the Creative Commons Attribution License (type CC-BY), which permits unrestricted use, distribution and reproduction in any medium, provided the original article is properly cited. 